\title{
OCULAR ISCHAEMIA
}

Ocular ischaemia occurs in a number of conditions which, although uncommon, are becoming increasingly recognized. Complete ischaemia of tissue is generally characterized by pain, loss of function, oedema, and eventual necrosis, while incomplete ischaemia leads to less abrupt metabolic changes that may be compensated by developing collateral circulations. These ischaemic processes may be observed in the eye.

The blood flow in the ocular vessels may be interrupted by emboli, by diseases of the vessel walls, or by external pressure on the vessels. Examination of the eye will usually indicate the site of the occlusion, while the general condition of the patient will often help determine the aetiology of the occlusion. Ocular ischaemia may result from interference with any of the following groups of blood vessels:

\section{(1) Great Vessels of the Head}

Occlusions of the aorta and its branches have been divided into two major groups: the aortic arch syndrome occurring in elderly people as a result of atheroma (Fig. 1a) or syphilitic arteritis, and pulseless disease occurring in young people as a result of a non-infective arteritis. This group of diseases gives rise to an incomplete ocular ischaemia often presenting with ocular pain, flashes of light, redness of the eye, reduced visual acuity, field defects, and amaurosis fugax. Arterio-venous anastomoses may be observed around the disc and haemorrhages and micro-aneurysms may be scattered throughout the retina. New vessel formation may occur in the retina, iris, and angle of the anterior chamber, and iridoplegia and iris atrophy with flare and cells in the aqueous may be present. Pigment clumping may be seen in the retina (Fig. $1 b$ ) and ischaemic optic atrophy may occur, often presenting as a pale swelling of the disc. Cataract and secondary glaucoma may be late complications.

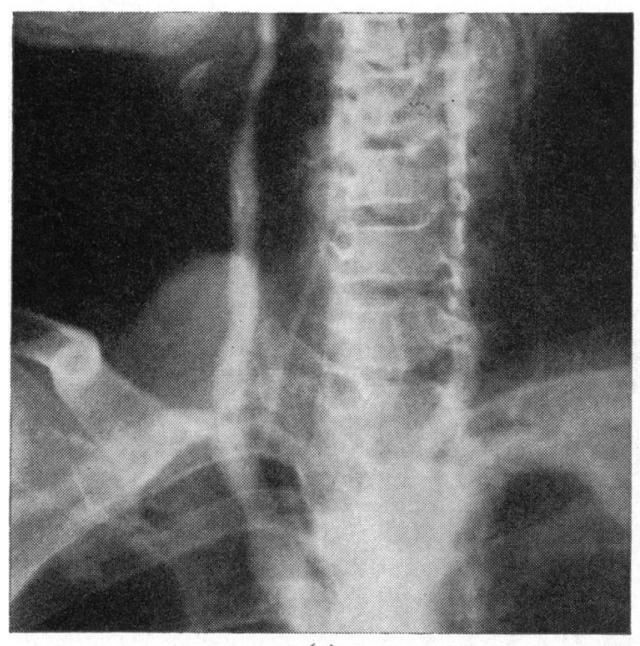

(a)

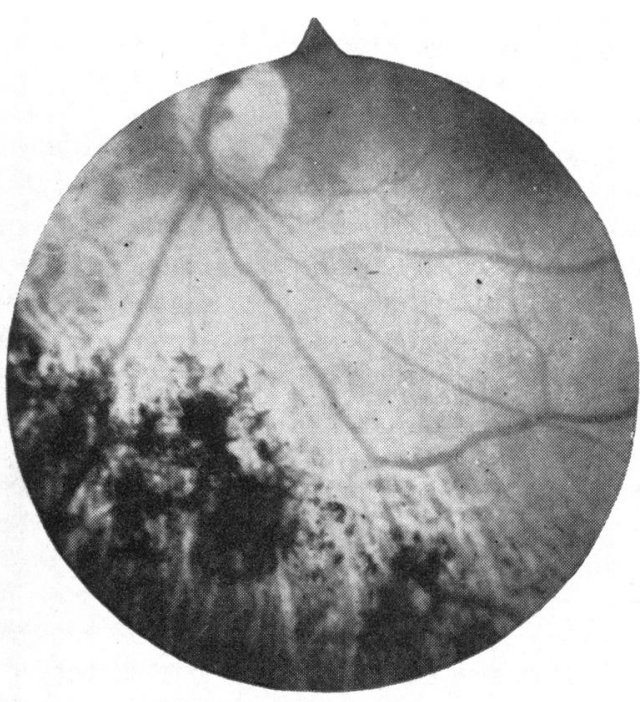

(b)

FIG. 1.-Gross obliterative disease of the major arteries in the neck. (a) Aortogram. (b) Left fundus showing pigmentary changes. This patient later developed the complete ocular ischaemic syndrome.

\section{(2) Ophthalmic Artery}

Blood flow in the ophthalmic artery may be reduced or interrupted by thrombosis, arterial disease, or a carotico-cavernous aneurysm and its surgical management. In this last condition the signs of ocular ischaemia - oedema of the retina and cotton-wool spots-may be overshadowed by those of increased venous pressure. If the aneurysm is treated surgically by the "trap" operation and clamping the ophthalmic artery, complete ocular ischaemia and blindness will result unless a 
collateral circulation via the external carotid artery is established. An insufficient collateral circulation may result in incomplete ocular ischaemia, with a pale oedematous disc and cotton-wool spots in the retina.

\section{(3) Central Artery of the Retina}

Occlusion of the central artery or its branches may result from emboli, arterial disease (atheroma or spasm), or increased orbital pressure. Emboli are commonly atheromatous in origin, appearing as bright plaques within the vessel, or may be composed of platelets derived from intra-arterial

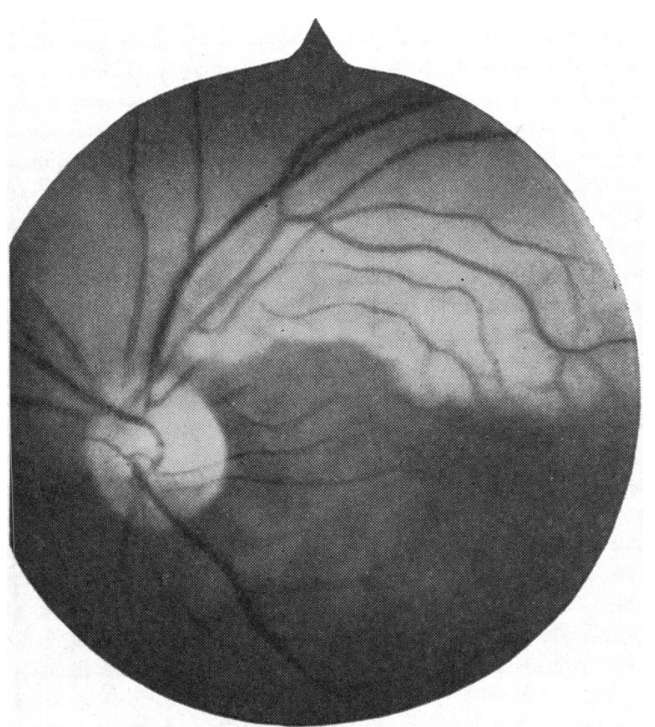

FIG. 2.-Recent occlusion of the superior branches of the central artery of the retina. thrombi. Arterial spasm may occur in migraine and quinine poisoning. Increased orbital pressure occurs in malignant exophthalmos, retro-ocular haemorrhage, or after excessive pressure bandaging.

The clinical picture of blindness or field defects with retinal oedema (Fig. 2) and a cherry red spot at the macula is well known. The retinal arteries may eventually become patent, although optic atrophy will ensue. Secondary glaucoma from rubeosis iridis may occur rarely. Arterial occlusion often accompanies venous thrombosis. Branch occlusion may eventually show signs of incomplete ischaemia with new vessel formation, reverse vessel flow, and condensation fibrosis leading to localized retinal detachment. Pigmentary disturbances in the retina, which often accompany incomplete ischaemia, are most commonly seen around the macula. Retinal ischaemia may be the underlying cause in the development of the retinopathies, the anoxia stimulating micro-aneurysmal formation with haemorrhages and exudates.

\section{(4) Short Posterior Ciliary Arteries}

These arteries may be occluded in giant cell arteritis and after orbital surgery. Pigmentary disturbances at the posterior pole have occurred both in these conditions and experimentally after division of these vessels, while acute necrosis of the posterior pole of the eye has been observed in giant cell arteritis.

\section{(5) Long Posterior Ciliary Arteries}

These arteries may be occluded by encircling procedures for retinal detachment resulting in the "string syndrome". This condition has a venous obstructive element, as shown by lid oedema, chemosis, and proptosis; but the pain, hypotension, fixed greenish iris with peripheral posterior synechiae, and the deep anterior chamber with a heavy flare are probably the result of arterial occlusion in the anterior segment of the eye.

The ocular response to complete ischaemia may be explained on general pathological principles, while that to incomplete ischaemia may suggest a basic aetiological factor in such varied conditions as pigmentary and vascular retinopathies, heterochromic cyclitis, and some forms of cataract.

\section{Illustrations:}

INSTITUTE OF OPHTHALMOLOGY, LONDON, W.C.1.
M. A. BedFord AND R. K. BLACH, INSTITUTE OF OPHTHALMOLOGY, LoNDON, W.C.1. 